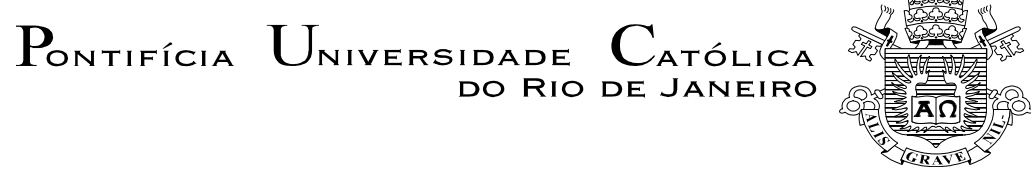

Nádia Maria Coelho Rodrigues

\title{
Concatenação Estatística de Dados e Afiliação
}

Estocástica

Tese apresentada ao Programa de Pós-Graduação em Engenharia Elétrica da PUC_Rio como requisito parcial para obtenção do título de Doutor em Engenharia Elétrica.

Orientador: Reinaldo C. Souza

Co-orientador: Kaizô I. Beltrão

Rio de Janeiro, 13 de outubro de 2009. 


$$
\text { Pontifícia Universidade } \text { Co }_{\text {datólica }}
$$

\title{
Nádia Maria Coelho Rodrigues
}

\section{Concatenação Estatística de Dados e Afiliação \\ Estocástica}

Tese apresentada como requisito parcial para obtenção do grau de Doutor pelo Programa de Pós-Graduação em Engenharia Elétrica do Departamento de Engenharia Elétrica do Centro Técnico Científico da PUC-Rio. Aprovada pela Comissão Examinadora abaixo assinada.

Prof. Reinaldo C. Souza

Orientador

Departamento de Engenharia Elétrica - PUC-Rio

\author{
Prof. Kaizô I. Beltrão \\ Co-Orientador \\ ENCE/IBGE
}

Profa. Monica Barros

Departamento de Engenharia Elétrica - PUC-Rio

Prof. Victor Hugo de Carvalho Gouvêa

UFF

Prof. Sérgio da Costa Cortes

Departamento de Informática - PUC-Rio

\author{
Profa. Ana Carolina Letichevsky \\ Fundação Cesgranrio \\ Prof.José Eugenio Leal \\ Coordenador Setorial do Centro \\ Técnico Científico
}

Rio de janeiro, 13 de outubro de 2009. 
Todos os direitos reservados. É proibida a reprodução total ou parcial do trabalho sem autorização da universidade, da autora e do orientador.

\section{Rodrigues, Nádia Maria Coelho}

$\mathrm{Na}$ Escola Nacional de Ciências Estatísticas graduou-se em Estatística em 1976 e no curso de mestrado em Estudos Populacionais e Pesquisas Sociais em 2003.

Ficha Catalográfica

Rodrigues, Nádia Maria Coelho

Concatenação estatística de dados e afiliação estocástica / Nádia Maria Coelho Rodrigues ; orientador: Reinaldo C. Souza ; coorientador: Kaizô I. Beltrão. - 2009.

$159 \mathrm{f}$; $30 \mathrm{~cm}$

Tese (Doutorado em Engenharia Elétrica)-Pontifícia Universidade Católica do Rio de Janeiro, Rio de Janeiro, 2009.

Inclui bibliografia

1. Engenharia elétrica - Teses. 2. Emparelhamento estatístico. 3. Suposição de independência condicional (CIA). 4. Informação auxiliar. 5. Procedimento hot deck. I. Souza, Reinaldo C. II. Beltrão, Kaizô I. III. Pontifícia Universidade Católica do Rio de Janeiro. Departamento de Engenharia Elétrica. IV. Título. 
A meus pais e aos meus filhos por seu amor, carinho e seu contínuo apoio e estímulo. 


\section{Agradecimentos}

Aos meus orientadores Professores Reinaldo C. Souza e Kaizô I. Beltrão, pelo apoio, confiança e pela sua dedicada orientação.

Ao professores examinadores Ana Carolina Letichevsky, Monica Barros, Sérgio da Costa Cortes e Victor Hugo de Carvalho Gouvêa.

Ao IBGE pelo apoio financeiro. Aos funcionários e colegas do IBGE, principalmente da Diretoria de Informática.

À Pontifícia Universidade Católica de Rio de Janeiro, a todos os professores e funcionários do Departamento.

A meus amigos Mário Gama e Emilia Matos por contínuo seu apoio e colaboração.

A todos os amigos e familiares que de uma forma ou de outra me estimularam ou ajudaram. 


\section{Resumo}

Rodrigues, Nádia Maria Coelho; Souza, Reinaldo C.. Concatenação

Estatística de Dados e Afiliação Estocástica. Rio de Janeiro, 2009. 159p. Tese de Doutorado - Departamento de Engenharia Elétrica, Pontifícia Universidade Católica do Rio de Janeiro.

O emparelhamento estatístico é a técnica de combinar informações de duas ou mais fontes de dados, possivelmente de pesquisas independentes, que possuam um subconjunto comum de variáveis, para produzir uma informação mais abrangente e coerente, em um arquivo de dados síntese, onde as variáveis observadas nas diferentes amostras são gravadas conjuntamente. Métodos computacionalmente intensivos viabilizam novas formas de emparelhamento estatístico. Em um estudo de simulação, um único arquivo de dados da PNAD é dividido em duas bases de dados para emular um caso de amostragem matricial. Esses dois arquivos são emparelhados estatisticamente utilizando quatro metodologias, e os resultados das mesmas são comparados com as do arquivo único original. A CIA (conditional independence assumption) não parece ser válida. Para evitar a suposição de independência condicional (CIA), os três métodos de emparelhamento estatístico desenvolvidos são baseados na hipótese do relacionamento teórico de afiliação estocástica entre as variáveis contínuas renda e aluguel, e dois deles usam também a informação de resíduos. Os métodos são comparados entre si e com o método clássico, que se baseia na suposição de independência condicional - CIA. Em uma abordagem não-paramétrica, com um objetivo micro, os métodos de emparelhamento estatístico propostos são irrestritos, e realizam-se em classes, definidas pela variável de número de cômodos. Usam um procedimento distance hot deck, além de adicionar os resíduos supostos conhecidos. Esse estudo investiga os resultados de viés e do $\mathrm{EQM}$, dos quatro métodos, investigando a preservação da correlação original entre renda e aluguel.

\section{Palavras-chave}

Emparelhamento estatístico, suposição de independência condicional (CIA), informação auxiliar, procedimento hot deck 


\section{Abstract}

Rodrigues, Nádia Maria Coelho; Souza, Reinaldo C.. Statistical Matching using Stochastic Affiliation. Rio de Janeiro, 2009. 159p. DSc Thesis - Departamento deEngenharia Elétrica, Pontifícia Universidade Católica do Rio de Janeiro.

Statistical matching is the art of combining information from two or more datasets, collected in independent samples, but a subset of the variables is common to both samples, to produce both coherent and comprehensive information in a synthetic data file, where variables observed in different samples are jointly recorded. Modern computing can make possible, under techniques described here, an advance in the application of Statistical Matching. It is reported on a simulation that splits a single file into two pieces, statistically matched the pieces using four methodologies and compares the results to the original single file. The Conditional Independence Assumption (CIA) did not seem a valid assumption. To avoid CIA, it is suggested two methods of statistical matching where kind of auxiliary information, based on Stochastic Affiliation relationship between income and rent, and residuals are used, in conjunction with nonparametric regression and hot deck distance. Both are compared not only with the classic method based on the CIA and regression without residuals but also with each other. In a nonparametric approach, with a micro objective, an unconstrained statistical matching is applied using hot-deck nearest neighbor within classes, using the household logarithm income and logarithm rent percentile groups. We are satisfied with the performance of creating a correlation coefficient of $Y$ and $Z$, as measured using MSE.

\section{Keywords}

Statistical matching, conditional independence assumption (CIA), auxiliary information, hot deck imputation procedure. 


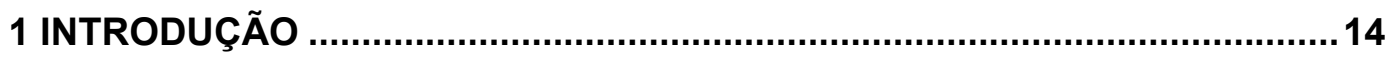

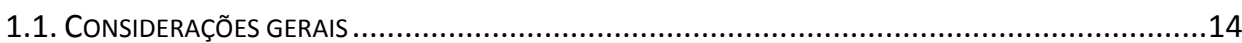

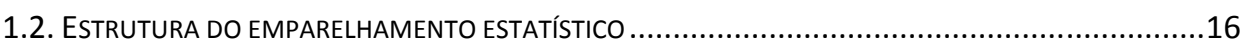

1.3. OBJETIVO, ABORDAGEM E REPRESENTATIVIDADE DO EMPARELHAMENTO ESTATÍSTICO.................20

1.3.1. Objetivo e abordagem do emparelhamento estatístico ...................................20

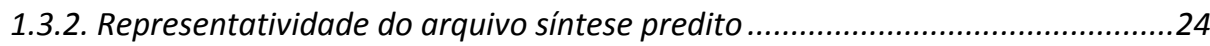

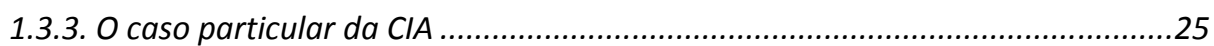

1.4. VARIANTE IMPORTANTE DO EMPARELHAMENTO ESTATÍSTICO ..............................................29

1.4.1. Aplicações de Amostragem Matricial...........................................................30

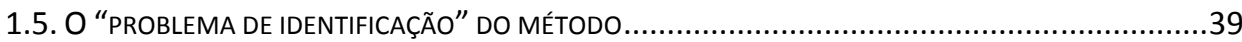

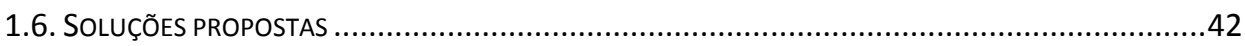

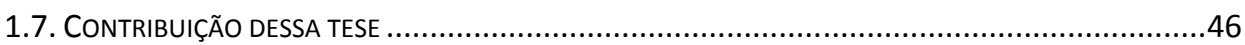

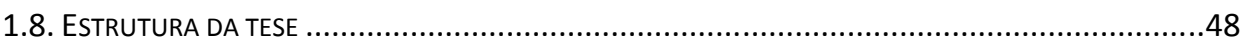

\section{REVISÃO DA LITERATURA SOBRE EMPARELHAMENTO ESTATÍSTICO 50}

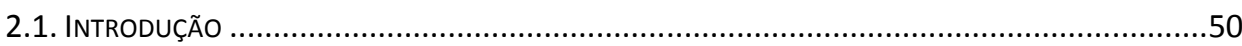

2.2. EMPARELHAMENTO ESTATÍSTICO RESTRITO E IRRESTRITO ...................................................56

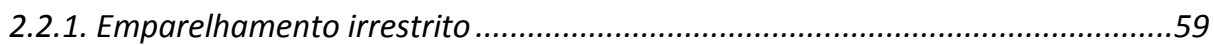

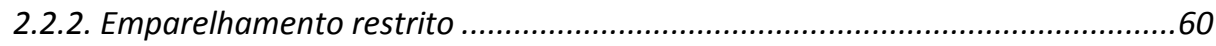

2.2.3. Comparação do emparelhamento irrestrito e restrito .....................................64

2.3. HARMONIZAÇÃO DAS PESQUISAS ANTES DO EMPARELHAMENTO .........................................65

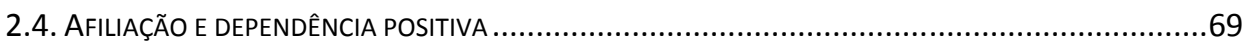

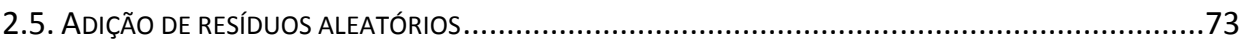

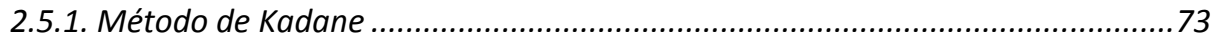

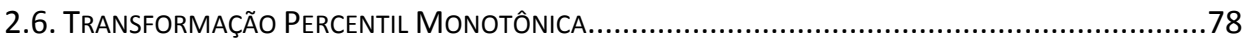

3 PRESSUPOSTO DE INDEPENDÊNCIA CONDICIONAL - CIA ......................79

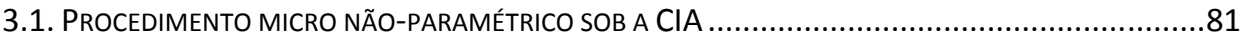

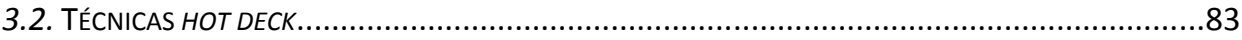

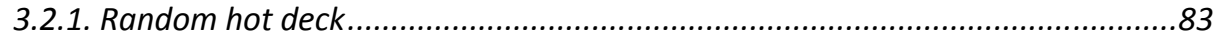

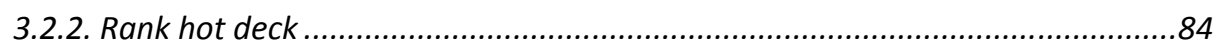

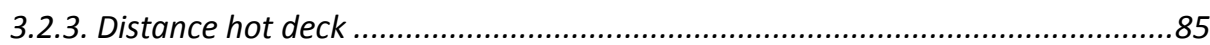

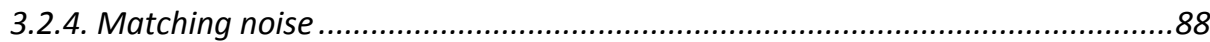

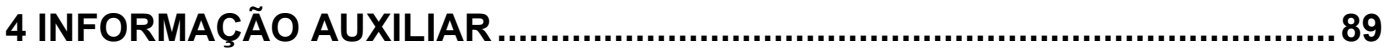

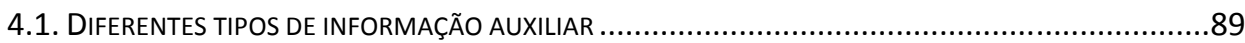

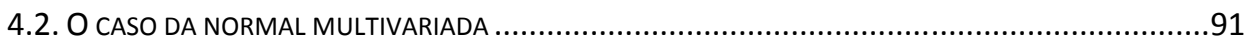


COVARIÂNCIAS MARGINAIS CONHECIDAS

4.3. PROCEDIMENTO MICRO NÃO-PARAMÉTRICO USANDO INFORMAÇÃO AUXILIAR .........................94

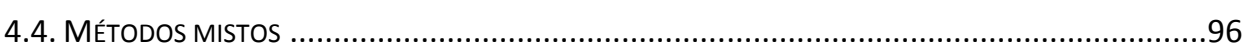

5 MÉTODO PROPOSTO E SIMULAÇÃO REALIZADA.....................................97

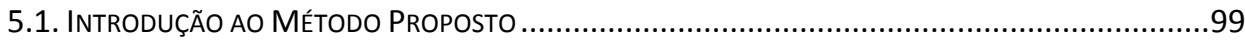

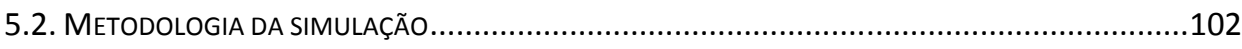

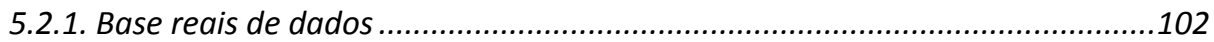

5.2.2. Propostas de procedimentos não-paramétricos de emparelhamento estatístico

107

6 ESTUDO DA SIMULAÇÃO...................................................................114

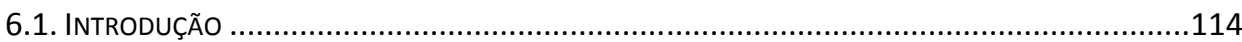

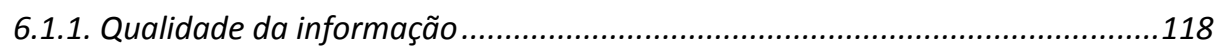

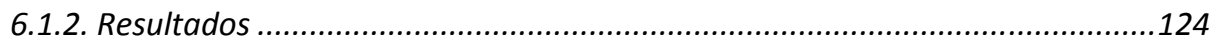

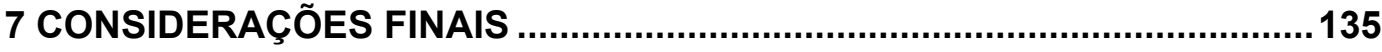

8 REFERÊNCIAS BIBLIOGRÁFICAS .........................................................138

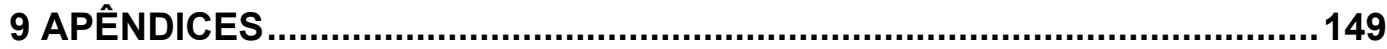

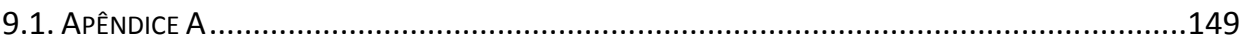

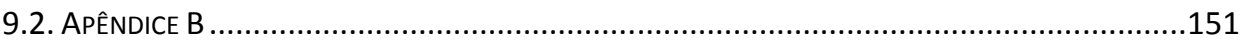

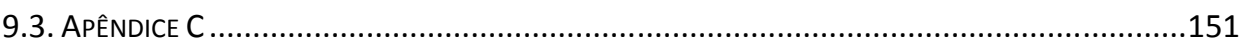

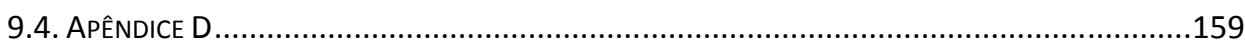




\section{Lista de Figuras}

Figura 1 - Problema geral: duas amostras independentes com $n_{A}$ e $n_{B}$ unidades, com suas variáveis especificas $Y$ e $Z$, com variáveis comuns $X$.................................................17

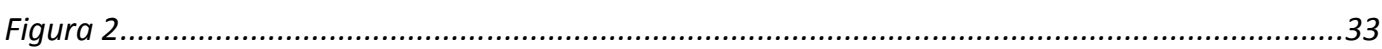

Figura 3 - Box-plot Lrenda, Laluguel, $p_{Y}$ e $p_{Z}$ por número de cômodos................................106

Figura 4 - Distribuição uniforme bidimensional dos resíduos, limitados pelas retas que garantem

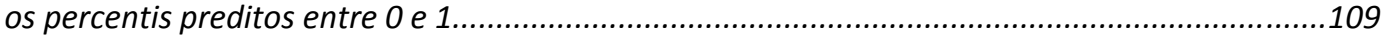

Figura 5 - Histograma e Box-plot da distribuição $(Y, Z)$ e seus percentis..................................120

Figura 6 - Distribuição uniforme bidimensional dos percentis $p_{Y}$ e $p_{Z}$, sem "indicação de ajuste" linear. .120

Figura 7 - Distribuição das variáveis da Lrenda e Laluguel por número de cômodos....................121

Figura 8 - Distribuição das variáveis da renda e aluguel e seus percentis...................................122

Figura 9 - Resíduos por número de cômodos...........................................................................122

Figura 10 - Distribuição de renda e aluguel para domicílios de 5 cômodos. .................................124

Figura 11 - Distribuição do aluguel antes e depois do emparelhamento ...................................133

Figura 12 - Distribuição do aluguel antes e depois do emparelhamento ..................................134 


\section{Lista de Tabelas}

Tabela 1: Registros do arquivo $A$

Tabela 2: Estatísticas descritivas de $A$

Tabela 3: Registros do arquivo $B$ .58

Tabela 4: Estatísticas descritivas de B .58

Tabela 5: Resultado do emparelhamento irrestrito. ..........................................................59

Tabela 6: Estatísticas descritivas do emparelhamento irrestrito $B$ doador ................................60

Tabela 7: Resultado do emparelhamento restrito...................................................................62

Tabela 8 - Número de registros nas amostras, dos arquivos, por número de cômodos ................123

Tabela 9 - Freqüência de Lrenda e Laluguel para domicílios de 5 cômodos.................................123

Tabela 10 - Valores originais das covariâncias e correlações.......................................................125

Tabela 11 - Estimativas das covariâncias do emparelhamento estatístico segundo os diferentes métodos.

Tabela 12 - Estimativas das correlações do emparelhamento estatístico segundo os diferentes métodos.

Tabela 13 - Estimativas das correlações do emparelhamento estatístico B doador. 159

Tabela 14 - Estimativas das correlações do emparelhamento estatístico A doador. 159 


\section{Lista de Quadros}

Quadro 1 - Abordagens e objetivos do emparelhamento estatístico. .........................................22

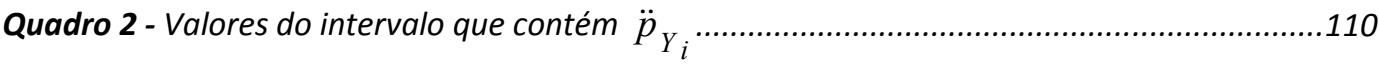




\section{Lista de Siglas}

\begin{tabular}{|c|c|}
\hline EQM & Erro quadrado médio \\
\hline IBGE & Instituto Brasileiro de Geografia e Estatística \\
\hline i.i.d & Independente e identicamente distribuídos \\
\hline MEC & Ministério de Educação e Cultura \\
\hline MRJ & Método de replicação Jackknife \\
\hline NAEP & National Center for Education Statistics \\
\hline NHIS & National Health Interview Survey \\
\hline PNAD & Pesquisa Nacional por Amostra de Domicílios \\
\hline POF & Pesquisa de Orçamentos Familiares \\
\hline SAEB & Sistema Nacional de Avaliação de Educação Básica \\
\hline INEP & $\begin{array}{l}\text { Instituto Nacional de Estudos e Pesquisas Educacionais Anísio } \\
\text { Teixeira }\end{array}$ \\
\hline PPV & Pesquisa sobre Padrões de Vida \\
\hline CIA & conditional independence assumption \\
\hline SAM & Social Accounting Matrix \\
\hline TNRIr & Transformação não-paramétrica relacional intervalar \\
\hline TNRIo & Transformação não-paramétrica relacional com interpolação \\
\hline EMV & Estimador de máxima verossimilhança \\
\hline SQS & Split Questionnaire Surveys \\
\hline PLRD & positive likehood ratio dependence \\
\hline
\end{tabular}

